\title{
THE HUMAN EAR RECOGNITION BASED ON PHASE- BASED MATCHING ALGORITHM
}

Muthukumar Arunachalam

Department of Electronics and Communication Engineering Kalasalingam Academy of Research and Education. Krishnankoil, (India). E-mail: muthuece.eng@gmail.com ORCID: https://orcid.org/0000-0001-8070-3475

\section{Citación sugerida:}

Arunachalam, M. (2020). The human ear recognition based on phase-based matching algorithm. 3C Tecnología. Glosas de innovación aplicadas a la pyme. Edición Especial, Marzo 2020, 141-157. http://doi. org/10.17993/3ctecno.2020.specialissue4.141-157

\section{Suggested citation:}

Arunachalam, M. (2020). The human ear recognition based on phase-based matching algorithm. 3C Tecnología. Glosas de innovación aplicadas a la pyme. Edición Especial, Marzo 2020, 141-157. http://doi. org/10.17993/3ctecno.2020.specialissue4.141-157 


\section{ABSTRACT}

In this modern era, biometric play a vital role in authentication process for an individual identification. Broadly biometrics has been classified into anatomical and behavioral characteristics. Among the many biometrics, this paper focus on new emerging biometric named as Ear biometrics. In this paper, Phase-only Correlation (POC) and Band-limited Phase Only Correlation (BLPOG) has been proposed for ear recognition. The phase information has been obtained by calculating similarity between input and query ear image using 2D-Discrete Fourier Transform (DFT) and auto correlation function. Finally the phase-based on image matching have being success implemented for human ear recognition endeavor. The experimental resultant effect of proposed algorithm has been performed using IIT Delhi ear database.

\section{KEYWORDS}

DFT, Phase Only Correlation (POG), Band-Limited Phase Only Correlation (BLPOC). 


\section{INTRODUCTION}

In impressive of last few years, Human Ear recognition has been becoming a very attractive in biometric authentication. The important reasons behind human ear biometric over other biometric modalities are smaller in size, very stable shape has proven by clinical observation (Rutty, Abbas, \& Crossling, 2005), uniform colors, and does not affected by any expression like face or changes in age or position or rotation (Malathy, Annapurani, \& Sadiq, 2013). The ear is a passive biometric as it can easily be captured from far distance, even if the person is not fully cooperative. Hence ear recognition is much interest for the researchers dealing with numerous applications in commercial and governmental, such as law enforcement, security systems and forensics (Jain, Ross, \& Pankanti, 2006). Therefore, ear biometric is a deserved preference for it providing a fine avail between cost and accuracy.

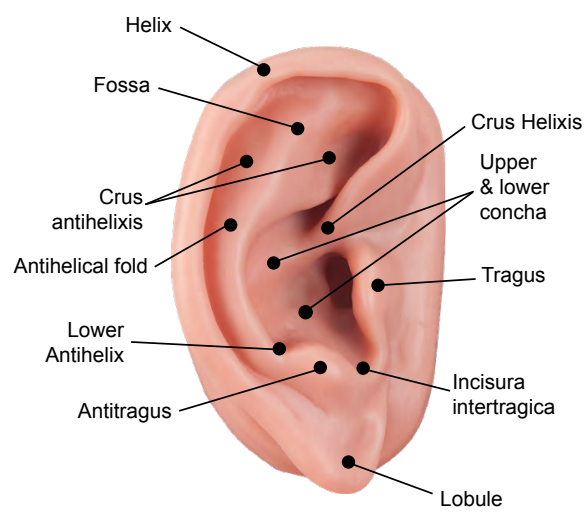

Figure 1. Ear Image.

The Figure 1 shows the ear image of an individual. It is stored in the IIT Delhi database and can be used for the task of identification through its unique characteristics. The human outer ears parts are formed by various terminologies include Helix, the Lobe, Antihelix, the tragus, concha along with an antitragus.

Recently, several matching algorithms have been adapted for the ear recognition process. Anyhow, these algorithms possess various limitations which are explained in this section. Primarily, the holistic matching method utilized from global features to extract the complete ear. Bustard et al. arrange the ear dataset by considering the planar surface. It was registered as homography transform designed of Scale Invariant Feature Transform from trails 
(Bustard \& Nixon, 2010). This method was strong to conditions in the appearance of pose variation and occlusion.

Ultimately, the matching algorithms with local feature have been adopted for identification. So Nanni et al. determined to decrease the dimension by usual sub-window of feature extraction with a Gabor Filter along with LaplacianEigenmaps (Zhu, Jia, \& Liu, 2009). They also used the Sequential Forward Floating Selection to sort out the finest matching feature with concord trained. However the function of Laplacian Eigen maps could drop datas extremely. Yazdanpanah et al. designed a region of covariance matrix through wellorganized plus strong ear caption, which is strong to clarification with fake difference (Miyazawa, Ito, Aoki, Kobayashi, \& Katsumata, 2006). In the following year, Yuan et al. ear region has extracted to segment by conserving from neighborhood impact and the particular region has chosen by the recognition rate (Zhang, Zhang, \& Zhang, 2009). This method could keep away from the occlusion problem other than shortcoming from multifake problem.
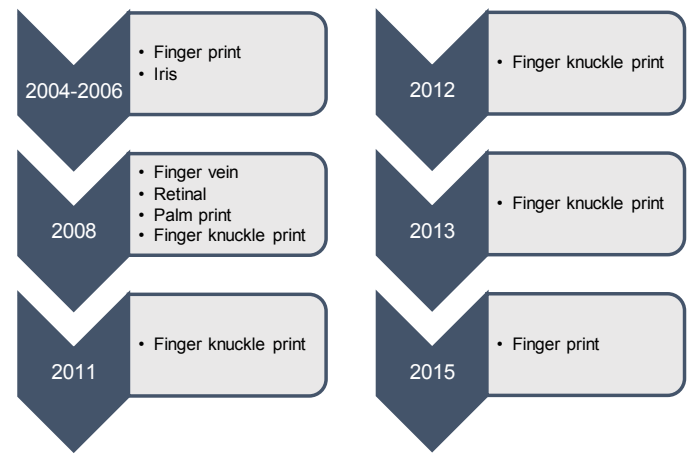

Figure 2. Different biometrics images used by POC and BLPOC.

The POC and BLPOC matching algorithm play the major role in image processing or pattern matching. There are many benefits in using Phase based matching algorithm such as simple to construct score, easy to implement, finest for multiple test and close to realtime performance. The Figure 2 shows different biometrics images are used by POC and BLPOC algorithm over the years for instance finger vein, finger print, iris, retinal, palm print as well as finger knuckle print except ear biometric. Therefore, this paper proposes human ear recognition based on phase-base matching algorithm. 


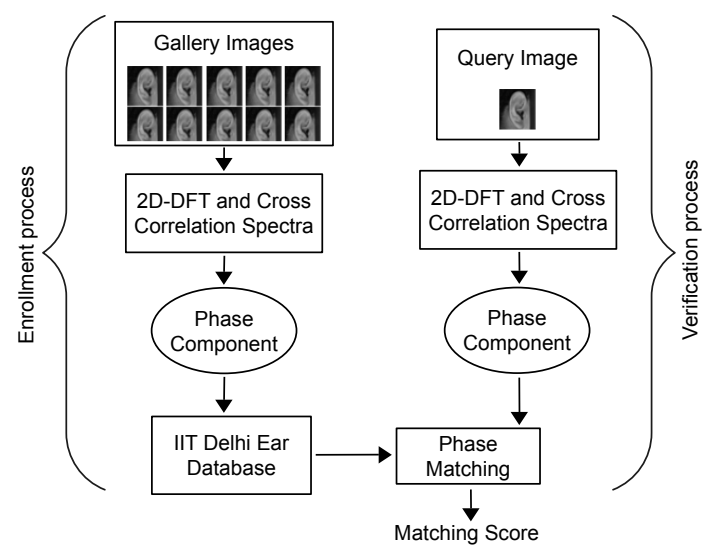

Figure 3. Proposed system.

The Figure 3 shows the proposed system of this paper. This proposed system involves two processes namely (i) Enrollment process and (ii) Verification process. First, enrollment process carries out the process that involves feature extraction of POC and BLPOC technique. This feature extraction minimizes the ear images into mathematical phase information. This information will be stored in database to establish the authentication of ear images. At the same time, verification process also arrive the same process for extracting features of POC and BLPOC technique which generates the phase information. This feature extraction converts the ear images into mathematical phase information. These both phase information's are afforded by decision making subsidiary. The decision making subsidiary used to provide a final conclusion (i.e. genuine or imposter output). The output of decision making is a potential matching score by matching identities.

The remainders in this paper are structured as follows: The second section discusses about survey of the literature which gives brief discussion about existed POG and BLPOC. The third section outlines the proposed work and its implementation of Human Ear Recognition. The fourth section discusses about presents the experimental results of proposed system. The conclusion has been described in the section 5 .

\section{RELATED WORK}

In this related work, discusses the detailed functions of phase-base on image matching algorithms (i.e. POG and BLPOC). The POC has been designed to find the correlation 
within two images (i.e. different images or similar images). The purpose of POC is to frame-up only phase feature and to isolate the magnitude feature of images for accuracy in image matching. During the trial time of POC with two similar images provides distinct sharp correlation peak and the correlation peak drops when two images are different (Takita, Muquit, Aoki, \& Higuchi, 2004). The experimental results of phase correlation demonstrate high robustness and accuracy in the pattern matching and the image registration (Ito, Nakajima, Kobayashi, Aoki, \& Higuchi, 2004). This has been achieved with direct estimation in Fourier domain through 2D phase difference data set (Miyazawa et al., 2006). This kind of POG correlation is more convenient and more profitable than other conventional correlation. The most remarkable advantages of POC are it provides better discrimination for similar images and high accuracy translational of displacement among the illustrations (Nanni \& Lumini, 2007). But for most part of phase function algorithm cannot carry the nonlinear deformation of illustrations (Rutty et al., 2005). The BLPOC is very crucial method for correlation in optimum band limit for phase based recognition. In POC function, every frequency components are concerned. Though, high frequency components tend to highlight and it probably prone to noise. Hence, worthless high frequency components are eliminated by setting a band-limited while calculating the cross-power spectrum (Yazdanpanah \& Faez, 2010). Also, in order to carry the nonlinear deformation, it employs BLPOC matching algorithm. The BLPOG be sufficient to estimate accurate overall correlation (Yuan, Wang, \& Mu, 2010). The modified version of POG is BLPOG which is committed to evaluate match between images due to its better performance than the POC function (Zhang, Zhang, Zhang, \& Guo, 2012). The proposed BLPOC perform (Zhang, Zhang, Zhang, \& Zhu, 2011) committed to biometric recognition tasks. Several authors attempted different modalities of biometric recognition by using POC and BLPOC are outlined in the given Table 1.

Table 1. Outline about Phase-Based Function matching algorithm in various biometrics.

\begin{tabular}{|c|c|c|}
\hline Author & Feature & Trait (Images) \\
\hline $\begin{array}{c}\text { (Takita et al., 2004). } \\
\text { (Ito et al., 2004). }\end{array}$ & POC and BLPOC & Finger Print \\
\hline $\begin{array}{c}\text { (Miyazawa, Ito, Aoki, } \\
\text { Kobayashi, \& Nakajima, } \\
\text { 2005). }\end{array}$ & POC and BLPOC & Iris \\
\hline (Zhu et al., 2009). & POC and BLPOC & Palm print \\
\hline
\end{tabular}




\begin{tabular}{|c|c|c|}
\hline Author & Feature & Trait (Images) \\
\hline $\begin{array}{c}\text { (Mahri, Suandi, \& Rosdi, } \\
\text { 2010). }\end{array}$ & POC and BLPOC & Finger Vein \\
\hline $\begin{array}{c}\text { (Miyazawa et al., 2006). } \\
\text { (Yuan et al., 2010). }\end{array}$ & POC and BLPOC & Retinal \\
\hline $\begin{array}{c}\text { (Zhang et al., 2011). } \\
\text { (Zhang et al., 2012). }\end{array}$ & POC and BLPOC & FKP \\
\hline $\begin{array}{c}\text { (Aoyama, Ito, \& Aoki, } \\
\text { 2014). }\end{array}$ & POC and BLPOC & FKP \\
\hline
\end{tabular}

Human Ear Recognition Using Phase-Based Function Matching.

\section{METHOD}

In this section, POC and BLPOC are discussed and derived in detail.

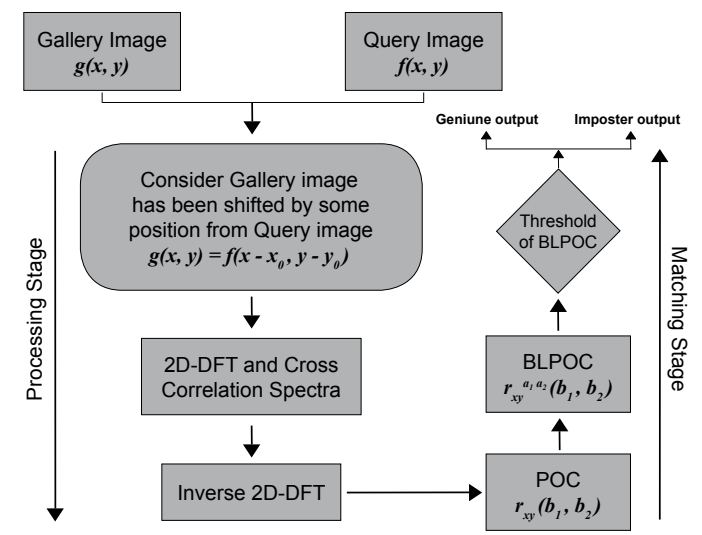

Figure 4. Overall structural outline of the proposed design of this paper.

The Figure 4 shows the overall structural outline of proposed design of this paper. This method is discussed in next section 3.1 and 3.2 in detail.

\subsection{PHASE-ONLY FUNCTION/CORRELATION (POC)}

This section discussed about the principal of POC. The POC is some time called as "PhaseOnly Function") (Zhang et al., 2009). Assume $2 \mathrm{M}$ x N images, g $x_{1}, x_{2}$ and the $f\left(x_{1}, x_{2}\right)$, where consider that the basis ranges are $x_{1}=M \ldots . . M(M \geq 0)$ and $x_{2}=N \ldots . . N(N \geq 0)$ in support of mathematical ease, and therefore $M=2 M+1$ and $N=2 N+1$. The conversation can be simply general towards non-nullifying basis ranges by rule of 2 image sizes. Let $F\left(a_{l}\right.$, 
$\left.a_{2}\right)\left(\right.$ and $G\left(a_{1}, a_{2}\right)$ denote the 2D-DFTs of $g\left(x_{1}, x_{2}\right)$ and, correspondingly. According to the description of DFT (Zhang et al., 2009), $F\left(a_{1}, a_{2}\right)$ and $G\left(a_{1}, a_{2}\right)$ be known through

$$
\begin{gathered}
F\left(a_{1}, a_{2}\right)=\sum_{x=0}^{M-1} \sum_{y=0}^{N-1} f\left(x_{1}, x_{2}\right) \cdot e^{\left(-j 2 \pi\left(\frac{a_{1} x_{1}}{M}+\frac{a_{2} x_{2}}{N}\right)\right)} \\
G\left(a_{1}, a_{2}\right)=\sum_{x=0}^{M-1} \sum_{y=0}^{N-1} g\left(x_{1}, x_{2}\right) \cdot e^{\left(-j 2 \pi\left(\frac{a_{1} x_{1}}{M}+\frac{a_{2} x_{2}}{N}\right)\right)}
\end{gathered}
$$

Consider $g\left(x_{1}, x_{2}\right)=f\left(x_{1}-x_{0}, x_{2}-y_{0}\right)$

Consider image is shifted by $x_{0}$ and $y_{0}$ portion, then $x_{0}=y_{0}=0$ the both is same. Now applying spatial shifting property,

$$
\begin{gathered}
F T\left[f\left(x_{1}-x_{0}, x_{2}-y_{0}\right)\right]=\exp ^{\left(-j 2 \pi\left(\frac{a_{1} x_{0}}{M}+\frac{a_{2} y_{0}}{N}\right)\right)} F\left(a_{1}, a_{2}\right) \\
\therefore G\left(a_{1}, a_{2}\right)=e^{\left(-j 2 \pi\left(\frac{a_{1} x_{0}}{M}+\frac{a_{2} y_{0}}{N}\right)\right)} F\left(a_{1}, a_{2}\right)
\end{gathered}
$$

For finding Correlation of, using equation

$$
f\left(x_{1}, x_{2}\right) * h\left(x_{1}, x_{2}\right)=\sum_{m=0}^{M-1} \sum_{n=0}^{N-1} f(m, n) \cdot h\left(x_{1}+m, x_{2}+n\right)
$$

Correlation phase spectra

$$
R_{G F}\left(a_{1}, a_{2}\right)=\frac{G\left(a_{1}, a_{2}\right) \cdot F^{*}\left(a_{1}, a_{2}\right)}{\left|G\left(a_{1}, a_{2}\right) \cdot F^{*}\left(a_{1}, a_{2}\right)\right|}
$$

Sub 6 and 7, so we get,

$$
\therefore R_{G F}\left(a_{1}, a_{2}\right)=\exp ^{\left(-j 2 \pi\left(\frac{a_{1} x_{0}}{M}+\frac{a_{2} y_{0}}{N}\right)\right)}
$$

2DIDFT-

$$
f\left(x_{1}, x_{2}\right)=\frac{1}{M N} \sum_{u=0}^{M-1} \sum_{v=0}^{N-1} F\left(a_{1}, a_{2}\right) \exp \left(-j 2 \pi\left(\frac{a_{1} x_{1}}{M}+\frac{a_{2} x_{2}}{N}\right)\right)
$$

Take 2D-IDFT for equation 7 , 


$$
\therefore r_{g f}\left(x_{1}, x_{2}\right)=\frac{1}{M N} \sum_{u=0}^{M-1} \sum_{v=0}^{N-1} R_{G F}\left(a_{1}, a_{2}\right) \exp \left(-j 2 \pi\left(\frac{a_{1} x_{1}}{M}+\frac{a_{2} x_{2}}{N}\right)\right)
$$

Put equation 8 in 10,

$$
\begin{gathered}
r_{g f}\left(x_{1}, x_{2}\right)=\frac{1}{M N} \sum_{u=0}^{M-1} \sum_{v=0}^{N-1} \exp ^{\left(-j 2 \pi\left(\frac{a_{1} x_{1}}{M}+\frac{a_{2} x_{2}}{N}\right)\right)} \exp \left(-j 2 \pi\left(\frac{a_{1} x_{0}}{M}+\frac{a_{2} y_{0}}{N}\right)\right) \\
=\frac{1}{M N} \sum_{u=0}^{M-1} \sum_{v=0}^{N-1} \exp ^{-j 2 \pi\left[\frac{a_{1} x_{0}}{M}+\frac{a_{2} y_{0}}{N}-\frac{a_{1} x_{1}}{M}-\frac{a_{2} x_{2}}{N}\right]} \\
r_{g f}\left(x_{1}, x_{2}\right)=\frac{1}{M N} \sum_{u=0}^{M-1} \sum_{v=0}^{N-1} \exp ^{\left.-j 2 \pi\left[\frac{a_{1}\left(x_{0}-x_{1}\right)}{M}+\frac{a_{2}\left(y_{0}-x_{2}\right)}{N}\right)\right)} \\
x_{0}-x_{1}=0 \quad y_{0}-x_{2}=0 \\
r_{g f}\left(x_{1}, x_{2}\right)=1 \quad(\text { Peak value })
\end{gathered}
$$

Respectively, where $a_{1}=-M_{1} \ldots M_{1}, \quad a_{2}=-M_{2} \ldots M_{2}, W_{N 1}=e^{-j 2 \pi / N 1} \quad, W_{N 2}=e^{-j 2 \pi / N 2}$ and $\Sigma_{n 1 n 2}$ denotes $\Sigma_{n_{1}=-m_{1}}^{M 1} \Sigma_{n_{2}=-m_{2}}^{M 2} . \quad A_{F}\left(a_{1}, a_{2}\right)$ and $A_{G}\left(a_{1}, a_{2}\right)$ are amplitude, and $\theta_{F}\left(a_{1}, a_{2}\right)$ and $\theta_{G}\left(a_{1}, a_{2}\right)$ are phase. The normalized cross power spectrum $R_{F G}\left(a_{1}, a_{2}\right)$ is set by

$$
R_{F G}\left(a_{1}, a_{2)}=\mathrm{F}\left(a_{1}, a_{2)} \overline{G\left(a_{1}, a_{2}\right)} / \mid \mathrm{F}\left(a_{1}, a_{2}\right) \overline{G\left(a_{1}, a_{2}\right)}\right.\right.
$$

Where $G\left(a_{1}, a_{2}\right)$ is the complex conjugate of $G\left(a_{1}, a_{2}\right)$ and $\theta\left(a_{1}, a_{2}\right)$ denotes the phase difference $\theta_{F}\left(a_{1}, a_{2}\right)-\theta_{G}\left(a_{1}, a_{2}\right)$. The POC function $r_{f g}\left(n_{1}, n_{2}\right)$ is the 2D IDFT of $R_{F G}\left(a_{1}, a_{2}\right)$ and is set by

$$
r_{f g}\left(x_{1}, x_{2}\right) \frac{1}{N_{1} N_{2}} \sum_{k 1 k 2} R_{F G}\left(a_{1}, a_{2}\right) W_{N 1}^{-a_{1} n_{1}} W_{N 2}^{-a_{1} n_{2}}
$$

Where $\Sigma_{a_{1}, a_{2}}$ denotes $\Sigma_{a_{1}=-m 1}^{M 1} \Sigma_{a_{2}=-m 2}^{M 2}$. When 2 images are alike, their POC perform provides a definite quick peak. When 2 images aren't alike, the height drop noticeably. The height of peak provides a decent likeness for match the image, with therefore the position of height shows change of location displacement among the illustration (Zhang et al., 2009). 


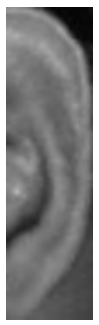

(a)

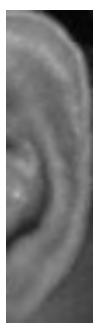

(b)

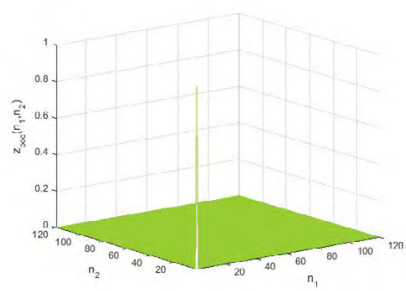

(c)

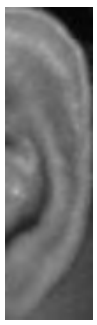

(d)

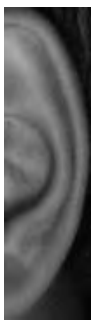

(e)

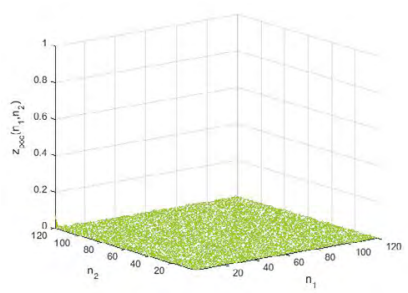

(f)

Figure 5. (a) Gallery image, (b) Query image, (c) POC for the same ear images, (d) Gallery image, (e) Query image and (f) POC for different ear images.

\subsection{BAND-LIMITED PHASE ONLY FUNCTION/CORRELATION (BLPOC)}

Consider to facilitate the range of the intrinsic frequency band be set through $a_{1}=-a_{1} \ldots$ $a_{1}$ and $a_{2}=-a_{2} \ldots a_{2}$, where $0 \leq a_{1} \leq M_{1}$ and $0 \leq a_{2} \leq M_{2}$. Therefore, the valuable size of frequency spectrum be set through $L_{1}=2 a_{1}+1$ and $L_{2}=2 a_{2}+1$ (Miyazawa et al., 2006). The BLPOG is known by means of

$$
r_{f g}^{a_{1}, a_{2}}\left(x_{1}, x_{2}\right) \frac{1}{L_{1} L_{2}} \sum_{a_{1}, a_{2}} \operatorname{Rg} f\left(a_{1}, a_{2}\right) W_{N 1}^{-a_{1} n_{1}} W_{N 2}^{-a_{2} n_{2}}
$$

Where $x_{1}=-a_{1} \ldots a_{1}, x_{2}=-a_{2} \ldots a_{2}$ and $\Sigma_{a_{1}, a_{2}}$ denote $\Sigma_{a=-a_{1}}^{a_{1}}$. Remind that the highest value of correlation peak of BLPOC is constantly normalized to 1 and it not lean on L1 and L2 (Nanni \& Lumini, 2007).

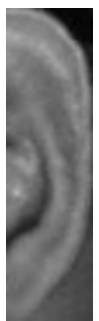

(a)

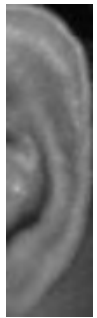

(b)

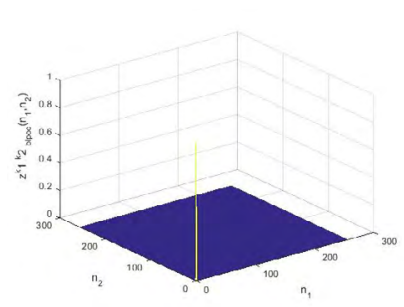

(c)

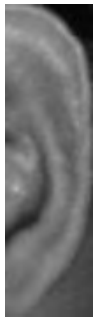

(d)

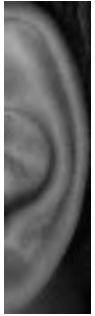

(e)

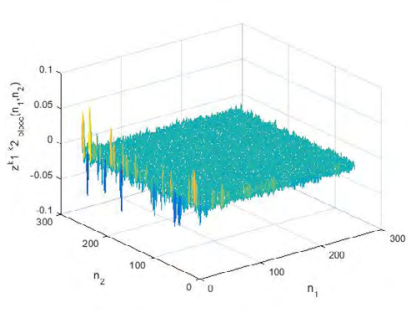

(f)

Figure 6. (a) Gallery image, (b) Query image, (c) BLPOC for the same ear images, (d) Gallery image, (e) Query image and (f) BLPOC for different ear images. 


\section{EXPERIMENT AND DISCUSSION}

The intended method be extensively investigated using an Ear images database provided by IIT Delhi. It is contained of the ear images, where collected from the students and staff at IIT Delhi (Zhu et al., 2009). In this paper proposed method are implemented and evaluated by using MATLAB software. Next in this paper proposed method has employee two processes: one is POC based phase matching components of two same images and two different images. Another one is to perform BLPOC based phase matching components of two same images and two different images. This issue has been showed in Figure 4 and 5 . The Figure 5 shows POC level estimated at 0.64, it express high matching trial has achieved. Uniformly Figure 6 shows BLPOC level estimated at 0.14, it express high matching trial has achieved. It shows better performance than other modalities that are previously existed.

\subsection{PERFORMANCE EVALUATION OF HUMAN EAR RECOGNITION}

The Receiver Operating Characteristic (ROC) curve evaluates the performance of the biometrics-based authentication system, which constructs with the help of False Reject Rate (FRR) and False Accept Rate (FAR) in various thresholds resting on matching score. Primarily, estimate the FRR in support of all the probable combinations number of authentic attempts. Similarly, estimate the FAR in support of all the probable combinations number of imposter attempts shown in Figure 7. In Figure 8, the ROC curve belongs to BLPOC; it demonstrates this algorithm is suitable for recognizing human ear images. Hence, proposed algorithm consider overall and confined deformation of human ear images together to calculate the scores of matching among the human ear images. Table 2 shows the EER [\%] and distance $\left(d^{\prime}\right)$ values of Ear identification.

$$
\mathbf{d}^{\prime}=\frac{\sqrt{2}\left|\mu_{\text {genuine }}-\mu_{\text {imposter }}\right|}{\sqrt{\sigma_{\text {genuine }}^{2}+\sigma_{\text {imposter }}^{2}}}
$$

Table 2. Equal error rate and distance (d') of the human ear recognition.

\begin{tabular}{|c|c|c|}
\hline Proposed algorithm & Equal error rate (\%) & Distance (d') \\
\hline BLPOC & 0.86 & 2.145 \\
\hline
\end{tabular}




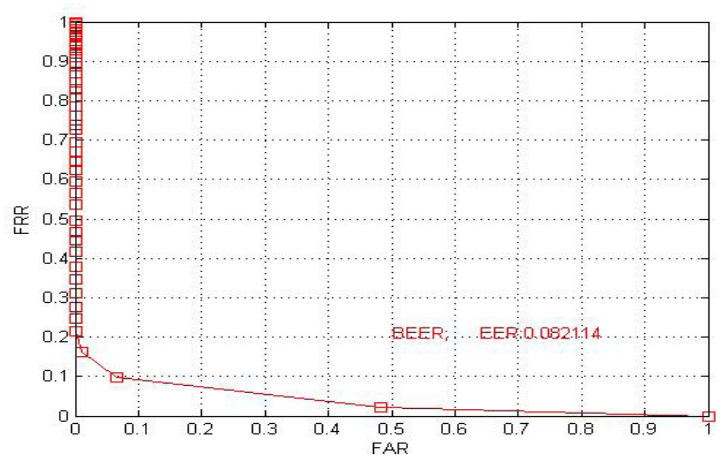

Figure 7. EER rate of proposed system.

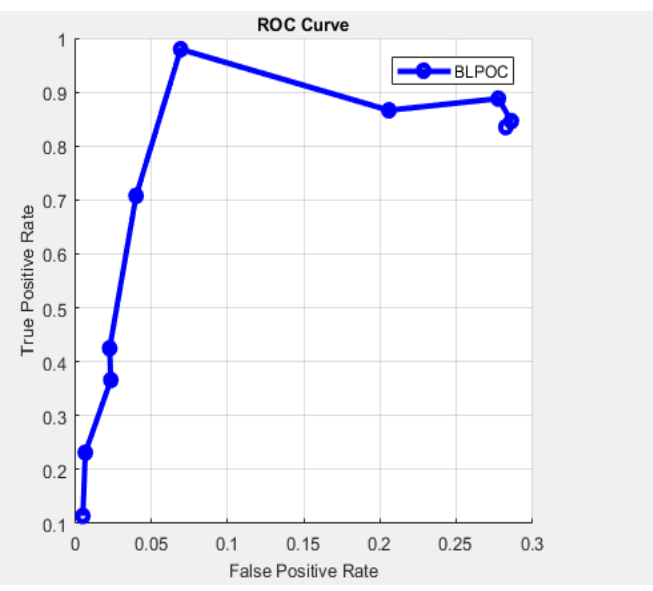

Figure 8. ROC curves for BLPOC algorithm.

Where, the mean value of genuine and imposter matching scores, respectively to an authentic and fraud identification, and the standard deviation of genuine and imposter matching scores, respectively to an authentic and fraud identification. The performances of a proposed method are able to assess in terms of identification accuracy, which is specified in the equation (17).

$$
\text { Accuracy }=100-\frac{\text { False rejection rate }+ \text { False acceptance rate }}{2}
$$




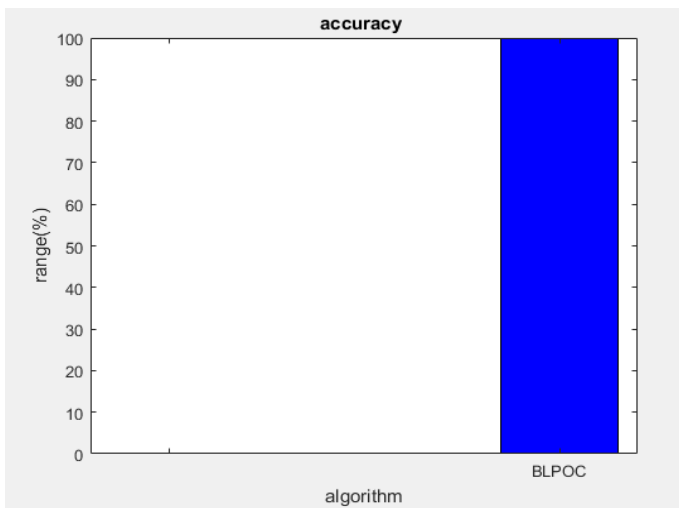

Figure 9. Accuracy of BLPOC algorithm.

The Figure 9 shows the accuracy of BLPOC algorithm. Where, FRR is the false rejection rate at which an authentic person is wrongly rejected as an imposter and FAR is the false acceptance rate, which denotes the rate of an imposter is wrongly accepted as an authentic person.

\section{CONCLUSION}

This paper intends a human ear identification using phase based function matching algorithm. The proposed BLPOC algorithm of Human Ear Recognition makes it possible to align ear images, correctly evaluated similarity between them and obtained the reliable matching score. The experimental results reveal that the intended method has achieved the well again Human Ear recognition performance and robustness over other previous methods. Extensively experiments were tested on the Ear database IIT Delhi. The proposed method of human ear recognition has performed the finest verification results on the Ear database IIT Delhi, with the equal error rate $0.86 \%$.

\section{ACKNOWLEDGEMENT}

I thank the department of Electronics and Communication Engineering of Kalasalingam University, (Kalasalingam Academy of Research and Education), Tamil Nadu, India for permitting to use the computational facilities available in Signal Processing and VLSI Design laboratory which was setup with the support of the Department of Science and Technology (DST). 


\section{REFERENCES}

Aoyama, S., Ito, K., \& Aoki, T. (2014). A finger-knuckle-print recognition algorithm using phase-based local block matching. Information Sciences, 268, 53-64. https://doi. org/10.1016/j.ins.2013.08.025

Bustard, J. D., \& Nixon, M. S. (2010). Toward unconstrained ear recognition from twodimensional images. IEEE transactions on systems, man, and cybernetics-Part A: Systems and Humans, 40(3), 486-494. https://doi.org/10.1109/TSMCA.2010.2041652

IIT Delhi Ear Database. (2007). www.iitdelhi/biometric/ear.html

Ito, K., Nakajima, H., Kobayashi, K., Aoki, T., \& Higuchi, T. (2004). A fingerprint matching algorithm using phase-only correlation. IEICE transactions on fundamentals of electronics, communications and computer sciences, 87(3), 682-691. http://www.aoki.ecei. tohoku.ac.jp/research/docs/e87-a_3_682.pdf

Jain, A.K., Ross, A., \& Pankanti, S. (2006). Biometrics: a tool for information security. IEEE transactions on information forensics and security, 1(2), 125-143. https://doi. org/10.1109/TIFS.2006.873653

Mahri, N., Suandi, S. A., \& Rosdi, B. A. (2010, August). Finger vein recognition algorithm using phase only correlation. In 2010 International Workshop on Emerging Techniques and Challenges for Hand-Based Biometrics (pp. 1-6). IEEE. https://doi.org/10.1109/ ETCHB.2010.5559283

Malathy, G., Annapurani, K., \& Sadiq, A. K. (2013). Analysis of fusion methods for ear biometrics. International fournal of Advanced Research in Computer Science and software Engineering, 3(1), 181-185.

Miyazawa, K., Ito, K., Aoki, T., Kobayashi, K., \& Katsumata, A. (2006, October). An iris recognition system using phase-based image matching. In 2006 International Conference on Image Processing (pp. 325-328). IEEE. https://doi.org/10.1109/ ICIP.2006.313159 
Miyazawa, K., Ito, K., Aoki, T., Kobayashi, K., \& Nakajima, H. (2005, September). An efficient iris recognition algorithm using phase-based image matching. In IEEE international conference on image processing 2005, (Vol. 2, pp. II-49). IEEE. doi: https://doi. org/10.1109/ICIP.2005.1529988

Nanni, L., \& Lumini, A. (2007). A multi-matcher for ear authentication. Pattern Recognition Letters, 28(16), 2219-2226. https://doi.org/10.1016/j.patrec.2007.07.004

Rutty, G. N., Abbas, A., \& Crossling, D. (2005). Could earprint identification be computerised? An illustrated proof of concept paper. International Journal of Legal Medicine, 119(6), 335-343. https://doi.org/10.1007/s00414-005-0527-y

Takita, K., Muquit, M. A., Aoki, T., \& Higuchi, T. (2004). A sub-pixel correspondence search technique for computer vision applications. IEICE transactions on fundamentals of electronics, communications and computer sciences, 87(8), 1913-1923. http://www.aoki.ecei. tohoku.ac.jp/research/docs/e87-a_8_1913.pdf

Yazdanpanah, A. P., \& Faez, K. (2010). Ear Recognition Using Bi-Orthogonal and Gabor Wavelet-Based Region Covariance Matrices. Applied Artificial Intelligence, 24(9), 863-879. https://doi.org/10.1080/08839514.2010.514228

Yuan, L., Wang, Z. H., \& Mu, Z. G. (2010, April). Ear recognition under partial occlusion based on neighborhood preserving embedding. In Biometric Technology for Human Identification VII (Vol. 7667, p. 766701). International Society for Optics and Photonics.

Zhang, L., Zhang, L., \& Zhang, D. (2009, September). Finger-knuckle-print verification based on band-limited phase-only correlation. In International Conference on Computer Analysis of Images and Patterns (pp. 141-148). Springer, Berlin, Heidelberg.

Zhang, L., Zhang, L., Zhang, D., \& Guo, Z. (2012). Phase congruency induced local features for finger-knuckle-print recognition. Pattern Recognition, 45(7), 2522-2531. https://doi.org/10.1016/j.patcog.2012.01.017

Zhang, L., Zhang, L., Zhang, D., \& Zhu, H. (2011). Ensemble of local and global information for finger-knuckle-print recognition. Pattern recognition, 44(9), 1990-1998. https://doi.org/10.1016/j.patcog.2010.06.007 
Zhu, Y. H., Jia, W., \& Liu, L. F. (2009, September). Palmprint recognition using bandlimited phase-only correlation and different representations. In International Conference on Intelligent Computing (pp. 270-277). Springer, Berlin, Heidelberg. 
